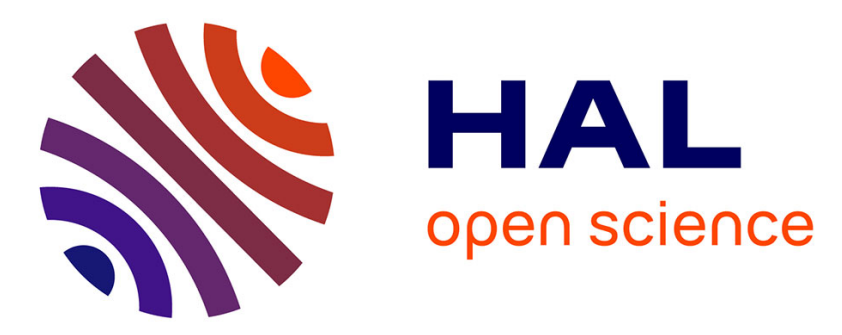

\title{
Spatial evolution of the plasma kernel produced by nanosecond discharges in air
}

Sergey Stepanyan, Nicolas Minesi, Augustin Tibère-Inglesse, Arthur Salmon, Gabi Daniel Stancu, Christophe O Laux

\section{- To cite this version:}

Sergey Stepanyan, Nicolas Minesi, Augustin Tibère-Inglesse, Arthur Salmon, Gabi Daniel Stancu, et al.. Spatial evolution of the plasma kernel produced by nanosecond discharges in air. Journal of Physics D: Applied Physics, 2019, 52 (29), pp.295203. 10.1088/1361-6463/ab1ba4 . hal-02118073

\section{HAL Id: hal-02118073 \\ https://hal.science/hal-02118073}

Submitted on 17 Jun 2019

HAL is a multi-disciplinary open access archive for the deposit and dissemination of scientific research documents, whether they are published or not. The documents may come from teaching and research institutions in France or abroad, or from public or private research centers.
L'archive ouverte pluridisciplinaire HAL, est destinée au dépôt et à la diffusion de documents scientifiques de niveau recherche, publiés ou non, émanant des établissements d'enseignement et de recherche français ou étrangers, des laboratoires publics ou privés. 


\title{
Spatial evolution of the plasma kernel produced by nanosecond discharges in air
}

\author{
S. Stepanyan, N. Minesi, A. Tibère-Inglesse, A. Salmon, G. D. Stancu and C.O. Laux \\ EM2C Laboratory, CNRS UPR288, CentraleSupélec, Université Paris Saclay, 3 rue Joliot-Curie, 91190 Gif- \\ sur-Yvette, France
}

\begin{abstract}
This work presents an experimental investigation of the hydrodynamic effects induced by nanosecond and conventional spark discharges. The energy deposited in sparks in the short breakdown time $(\sim 1 \mathrm{~mJ} / \mathrm{mm})$ induces hydrodynamic effects that redistribute the energy over a large volume $\left(\sim 1 \mathrm{~cm}^{3}\right)$ surrounding the initial plasma channel. This process influences the subsequent formation of the ignition kernel and the initiation of combustion. The experimental results presented in this paper were obtained with a set of synchronized diagnostics including Schlieren, $\mathrm{OH}$ Planar Laser Induced Fluorescence and electrical measurements of the energy deposited in the plasma. It is shown that the motion of the gas excited after the discharge breakdown depends not only on the total deposited energy but also on the dynamics of the energy input in the plasma. Finally, the effects of nanosecond sparks are compared with those of conventional sparks used for internal combustion engines. We show that, with 20 times less energy, the nanosecond spark produces a twice bigger excited gas volume than the conventional spark. This is because the energy deposited by the nanosecond spark during the breakdown stage is three times higher than for the conventional spark.
\end{abstract}

\section{Introduction}

The effect of spark discharges on the ignition of combustible mixtures has been the object of intense research for decades because of the complexity of the physico-chemical mechanisms involved [Matveev2013], [Maly 1984]. The theoretical description of plasma-assisted ignition (PAI) is particularly difficult because combustion chemistry must be coupled with plasma chemistry [Sher 1992], [Kossyi1992], [Starikovskiy2013], [Castela2016]. Moreover the kinetic mechanisms for plasma and combustion must also be coupled with electrodynamic and transport equations.

The action of a spark discharge on the formation of the ignition kernel is a combination of the following effects:

i) heat release, i.e. heating of the combustible mixture;

ii) chemical effects, i.e. production of active species, such as $\mathrm{O}, \mathrm{OH}$ etc.;

iii) hydrodynamic effects induced by the plasma and affecting the kernel formation.

Depending on the conditions, some of these effects can be dominant or negligible compared to the others. For example, the contribution of hydrodynamic effects is negligible in the case of ignition by low energy glow/corona discharges [Bozhenkov2003], [Wang2005], [Bellenoue2007], [Singleton2011], [Adamovich2014]. Moreover, in these references the electron-induced discharge chemistry can be separated from the combustion chemistry. The negligible role of hydrodynamic effects and the separation of combustion plasma/chemistry allow to simplify the theoretical description for these cases.

For sparks at higher pressures $(>1 \mathrm{~atm})$ the role of hydrodynamic effects cannot be neglected. This is demonstrated in several works [Olsen1954], [Kono1988], [Bane2014], [Lo2014], [Seydou2016], [Lovascio2017], [Xu2016]. Particularly, the results presented in [Olsen1954] demonstrate that a single submicrosecond spark of $245 \mathrm{~mJ}$ initiated in ambient air across a 1-mm gap produces a hydrodynamic distortion of the surrounding gas in a few tens of $\mu$ s. Olsen [Olsen 1954] also noted that the shape of the hot and excited gas volume produced by a single spark becomes toroidal (5-mm diameter) about $100 \mu$ s after the 
discharge. He concluded that the expansion of the gas excited by a spark is accompanied by circulation of the fresh gases incoming from the edges near the electrode tips. This gas motion was described by numerical models developed for conventional car sparks [Thiele2000], [Ekici2007], and more recently for nanosecond sparks [Castela2017]. Experimental confirmation of the recirculation phenomenon by particle image velocimetry can be found in [Seydou2016].

For discharges of nanosecond duration, heat and radical species (particularly atomic oxygen) are produced on nanosecond time scales via the so-called ultrafast heating and oxygen production process [Stancu2008], [Rusterholtz2013], [Popov2001]). The ultrafast heating process can induce hydrodynamic effects in the form of an above-mentioned recirculation zone and the creation of a torus of gas filled with radicals and heat [Castela2017], thus transporting the radicals and the heat into a volume much larger than the initial discharge channel. Therefore, this expansion spreads the initial plasma energy over larger gas volumes and thus is useful to enhance the combustion process.

However, there are still numerous questions regarding the hydrodynamic processes induced by nanosecond spark discharges. First, the creation of a torus appears to only occur above a certain threshold of energy deposited into the gas. Second, its creation also appears to be a function of the gap distance. Thus, the first goal of this paper is to investigate the conditions of creation of these toruses.

Another question is related to the dynamics of energy deposition. In particular, it is interesting to compare the hydrodynamic effects produced by the nanosecond sparks with those created by the conventional automotive sparks, where the energy is deposited over millisecond time scales. Maly et al [Maly1979] demonstrated that the conventional spark creates a cylindrical volume of active gas, which is entirely controlled by the breakdown stage and does not grow during the following arc and glow stages even though most of the energy is deposited in the plasma during these periods. Therefore, Maly et al claimed that in order to increase the active gas volume it is necessary to increase the energy deposited during the breakdown stage. Thus it is interesting to compare the nanosecond spark, where energy is deposited over a very short time, with the conventional spark.

Finally, nanosecond sparks are often applied in repetitive mode at high frequencies from 1 to $100 \mathrm{kHz}$ [Do2010, Starikovskiy2013, Lefkowitz2018]. It is therefore important to understand what is the effect of repetitively pulsed discharges on hydrodynamics. It was shown in [Stepanyan2017] that the volume of the gas excited by a burst of sparks is significantly larger than the volume excited by a single spark. Particularly, the gas volume excited by a burst of sparks [Stepanyan2017] with the total energy of $40 \mathrm{~mJ}$ has spatial scale of $15 \mathrm{~mm}$ while a single spark of larger energy ( $245 \mathrm{~mJ})$ [Olsen1954] excites a volume of 5 $\mathrm{mm}$ scale. The excitation of a larger gas volume is clearly favorable for ignition of lean or not premixed mixtures and for the stabilization of lean flames. It should be noted that the average energy density released in a burst of nanosecond sparks can provide a reliable ignition at considered conditions (see [Lovascio2017]).

Section 2 presents the experimental setup for the nanosecond discharges investigated in this work, along with the setup for optical and electrical diagnostics. Section 3 contains a brief description of nanosecond discharges used in this work. Section 4 presents results of our studies of the hydrodynamic effects induced by a single nanosecond spark in ambient air. Section 5 compares the hydrodynamic effects obtained with a single nanosecond discharge and a single conventional (millisecond) discharge. Finally, Section 6 examines the effects of multiple nanosecond discharges in ambient air. 


\section{Experimental setup}

Most of experiments presented here were performed with nanosecond sparks initiated by overvoltage pulses between pin electrodes. Some experiments were performed in pin-to-plane geometry. The pins were of conic shape with $100-\mu \mathrm{m}$ radius-of-curvature tips and were made of stainless steel. No noticeable difference was observed in the energy deposition or in the induced hydrodynamic effects [Stepanyan2017] for pin-electrodes with different radii of curvature. The distance between the electrodes was varied between 1 and $6 \mathrm{~mm}$. As the discharges were initiated at low frequency $(10 \mathrm{~Hz})$, no electrode erosion was observed under our conditions. In the case of the burst of a few tens of sparks at a few tens of $\mathrm{kHz}$ no erosion was observed either

Nanosecond spark discharges were initiated by a high-voltage generator (FID, FPG 30$100 \mathrm{MC} 4 \mathrm{~K})$ with pulses of 10 -ns duration, amplitudes up to $30 \mathrm{kV}$ and Pulse Repetition Frequencies (PRF) up to $100 \mathrm{kHz}$. The electrical characteristics of the discharge were monitored by high-voltage probes (Lecroy PPE) and a current probe (Pearson model 6585) installed in the middle of the 6-m cable $(75 \Omega)$ connecting the electrodes and the high-voltage generator. The signals obtained with the probes were recorded by high-frequency oscilloscope (Lecroy HDO6000). Figure 1 presents oscillograms typical for nanosecond sparks. Voltage and current curves were obtained for a single nanosecond spark initiated in ambient air by a $10-\mathrm{ns}$ pulse of $16 \mathrm{kV}$ in open circuit (which corresponds to $8 \mathrm{kV}$ in the middle of $75-\Omega$ cable where the electrical measurements were done). The distance between the electrodes was $1.5 \mathrm{~mm}$. The integrated product of voltage and current curves provides the evolution of the deposited energy. One can distinguish the following pulses: (1) incident to the electrodes, (2) reflected from the electrodes, (3) pulse reflected from generator and (4) reflected second time from the electrodes. The pulse (2) reflected from the electrodes produces the plasma. The energy deposited in plasma by the primary pulse is $2.7 \mathrm{~mJ}$, while the energy deposited in the secondary pulse is about $10 \%$ of this value. The detailed procedure for energy measurements for nanosecond discharges can be found elsewhere [Rusterholtz2013].

Hydrodynamic effects induced by nanosecond sparks were compared to those produced by a conventional AUDI system. The spark produced by AUDI system is a discharge with a breakdown stage similar to those presented in Figure 1 for a nanosecond spark. However, after the breakdown the voltage is still applied to the electrodes by the electrical circuit of AUID system during $2.5 \mathrm{~ms}$. Typical values of voltage and current in plasma during this period are $500 \mathrm{~V}$ and $0.1 \mathrm{~A}$ (see Figure 2 of [Lacour2016]).

The Schlieren experiments were performed with a light source (CAVILUX HF) providing laser light pulses of duration $100 \mathrm{~ns}$, wavelength centered at $640 \mathrm{~nm}$, and frequencies up to $900 \mathrm{kHz}$. The output of the light source was collimated with a 1-m focal length convex-plane lens. The beam, partly deflected in the discharge owing to refractive index gradients, was directed to a spherical mirror of 2-m focal length. A knife-edge was placed at the focal spot of the mirror to cut $80 \%$ of the light produced by the source. A second spherical mirror of 2$\mathrm{m}$ focal distance was placed between the knife-edge and the ICCD camera in order to reach a magnification factor of 1.8. A fast intensified camera (Photron SA-X2) was used to obtain Schlieren images or to map the spontaneous emission of the active species produced in the plasma. 


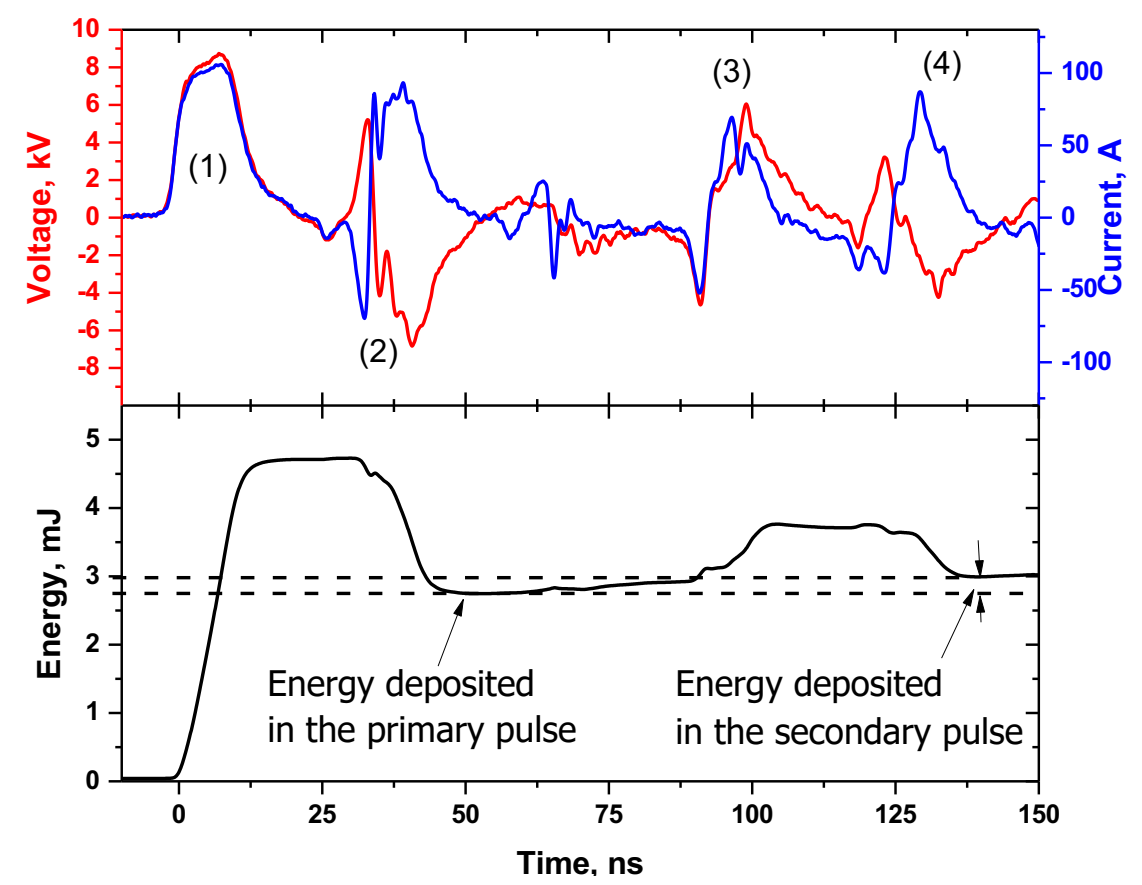

Figure 1. Typical oscillograms obtained for a nanosecond spark initiated in ambient air by a 10-ns pulse of $8 \mathrm{kV}$ ( $75 \mathrm{Ohm}$ cable). The interelectrode distance was $1.5 \mathrm{~mm}$. Figure on the top presents the voltage/current oscillograms and the figure on the bottom shows the evolution of the energy deposited in plasma.

The spatial distribution of active species such as $\mathrm{OH}$ produced in the discharge was measured by monitoring the PLIF intensity from the area corresponding to the discharge afterglow up to $200 \mu \mathrm{s}$ after the discharge. A frequency-doubled dye laser (Continuum ND6000) with Rhodamine 590 dye pumped by a Nd:YAG laser (Continuum Precision) at $532 \mathrm{~nm}$ was used to excite the $\mathrm{Q}_{1}(6)$ transition of $\mathrm{OH}\left[\left(\mathrm{X}^{2} \Pi_{\mathrm{i}}, v^{\prime \prime}=0\right) \rightarrow\left(\mathrm{A}^{2} \Sigma^{+}, v^{\prime \prime}=1\right)\right]$ at $282.935 \mathrm{~nm}$. The $\mathrm{OH}$ fluorescence signal was collected using a bandpass filter (CORION 10BPF10-310) centered at $310 \mathrm{~nm}$ with 11-nm FWHM placed before the ICCD camera (PiMax2, Princeton Instruments). The peak transmission of the filter was about $20 \%$. The PLIF laser sheet was formed by a combination of a cylindrical lens $(\mathrm{f}=15 \mathrm{~cm})$ and a spherical lens $(\mathrm{f}=10 \mathrm{~cm})$. The height of the sheet was $10 \mathrm{~mm}$ and its thickness was $0.2 \mathrm{~mm}$. The laser frequency was 10 $\mathrm{Hz}$, with a pulse FWHM of $6 \mathrm{~ns}$. The energy of the laser pulses used in experiments was $2 \mathrm{~mJ}$. Therefore the fluence of the laser sheet is about $0.1 \mathrm{MW} / \mathrm{mm}^{2}$.

The rotational temperature measurements from $\mathrm{N}_{2}\left(\mathrm{C}^{3} \Pi_{\mathrm{u}} \rightarrow \mathrm{B}^{3} \Pi_{\mathrm{g}}\right)$ OES and LIF spectra were obtained using a monochromator (Acton 500i) with a 2400 -groove/mm grating blazed at $240 \mathrm{~nm}$. The monochromator was coupled with an ICCD camera (PiMax2, Princeton Instruments). The OES was used in this work to control the discharge reproducibility and to confirm that the observed LIF images obtained in ambient humid air were due to $\mathrm{OH}$ fluorescence and not to $\mathrm{NO}$ or $\mathrm{N}_{2}$.

It should be noted that the nanosecond spark used in the present work was highly reproducible from shot to shot. Particularly the spontaneous emission of molecular nitrogen during the discharge did not change by more than $7 \%$ from shot to shot. The gas temperature variations extracted from spectra of the second negative system of nitrogen demonstrate that the temperature variations from shot to shot are below $50 \mathrm{~K}$ for temperatures higher than $1000 \mathrm{~K}$. The energy deposited in the plasma varied by less than $4 \%$ shot-to-shot. More details 
about the discharge reproducibility can be found in [Xu2013]. Finally such reproducibility allows to do sequences of phase-locked images of spontaneous emission, Schlieren and LIF.

The discharge, laser, and cameras were synchronized with a gate-and-delay generator (BNC Model 575). More details about synchronization can be found elsewhere [Pai2008], [Xu2013].

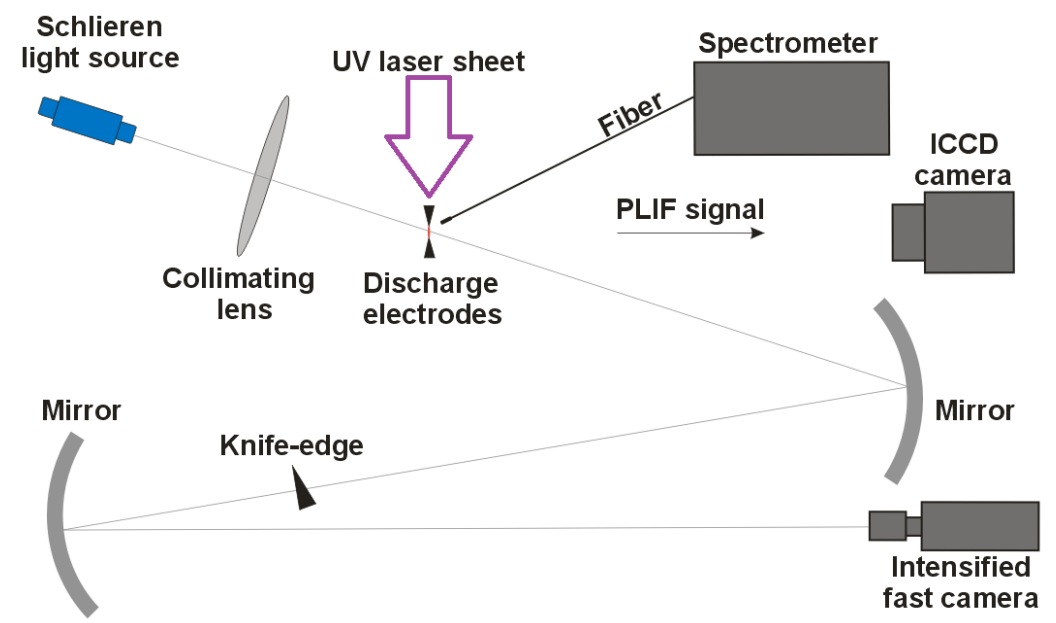

Figure 2. Optical diagnostics: PLIF, OES and Schlieren setup. The focal length of the lens used for Schlieren diagnostics is $1 \mathrm{~m}$. Both spherical mirrors have a focal distance of $2 \mathrm{~m}$.

\section{Discharge characterization}

The hydrodynamic effects studied in this work were induced by nanosecond sparks. The discharge was initiated by overvoltage pulses of 10-ns duration in ambient air between the electrodes separated by 1-6 mm. Detailed description of such discharges can be found elsewhere [Lo2014], [Stancu2008], [Rusterholtz2013], [Pai2010]. This section briefly presents the main characteristics of nanosecond sparks related to the induced hydrodynamic effects.

It has been shown in [Rusterholtz2013] that up to 35 percent of the discharge energy is spent for the oxygen dissociation. This dissociation is accompanied by a sharp rise of temperature for about $1000 \mathrm{~K}$ in $10 \mathrm{~ns}$. It has also been shown in [Popov2001] that the fractions of energy spent for the gas heating and for the oxygen dissociation have the same order for various interelectrode distances.

Such a sharp heat release in plasma induces a shock wave and further non-diffusive expansion of the hot gas filled by active species produced in the discharge (see [Olsen1954]). Atomic oxygen produced in plasma is converted into $\mathrm{OH}$ at nanosecond scale for the considered conditions [Stancu2008], i.e. before the gas expansion starts.

Finally one can say that the energy deposited in plasma is the main parameter driving the hydrodynamic effects induced by the spark and the production of chemically active species that can affect combustion. The data below demonstrate the results of the energy measurements for the conditions used in this work.

Table 1 presents the values of the total energy deposited in a spark initiated by a single 10ns pulse of $30-\mathrm{kV}$ amplitude (open circuit) for the various interelectrode gaps investigated here. The electrode potentials were $+15 \mathrm{kV}$ and $-15 \mathrm{kV}$. For the 4-mm gap, experiments were also conducted with a $400-\Omega$ resistance placed in series with the pin electrodes in order to investigate the effect of a reduced energy deposition. 


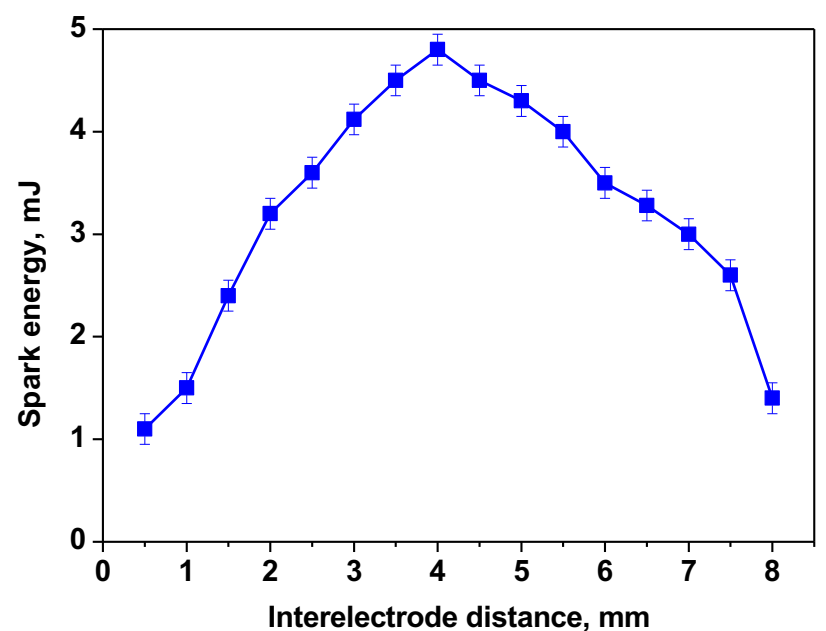

Figure 3. Dependence of the energy deposited in a single spark $v s$ interelectrode distance. The discharge was initiated in ambient air between pin electrodes by a $10-n$ s pulse of $30-\mathrm{kV}$ amplitude. No external resistance.

As can be seen from Table 1 and Figure 3, for gap distances between 0.5 and $4 \mathrm{~mm}$ the total deposited energy increases almost linearly from 1 to $5 \mathrm{~mJ}$. Then, at longer gap distances, the energy decreases. The energy deposited in the 6-mm gap is about the same as for the 2mm gap: this is due to different combinations of the reduced electric field and plasma volume. The two cases at 2 and $6 \mathrm{~mm}$ will provide a comparison of discharges with the same total deposited energy but different interelectrode distances. The data presented in Table 1 allow to select discharges with the same energy deposition per mm of spark (lineic energy) and discharges with the same total deposited energy but different lineic energy. We chose these cases to investigate whether the hydrodynamic motion induced by a discharge is governed by the lineic energy or by the total energy.

Table 1. Energy deposited in a single nanosecond pulse discharge for various interelectrode distances and resistances attached to the electrodes. In all cases, the applied voltage is fixed at $30 \mathrm{kV}$ (open circuit).

\begin{tabular}{|l|c|c|c|c|c|c|}
\hline Gap, $\mathrm{mm}$ & 1 & 2 & 3 & 4 & 4 & 6 \\
\hline External resistance, $\Omega$ & 0 & 0 & 0 & 0 & 400 & 0 \\
\hline Energy $E, \mathrm{~mJ}$ & 1.5 & 3.2 & 4.1 & 4.8 & 2.7 & 3.5 \\
\hline
\end{tabular}

\section{Hydrodynamic effects induced by a single nanosecond spark}

The high reproducibility of the single-pulse nanosecond sparks at ambient conditions makes it possible to analyze the spark events with phase-locked PLIF and Schlieren images. The hydrodynamic effects induced by a single nanosecond spark at the considered conditions are also reproducible up to the millisecond time scale. Several OH PLIF images were thus obtained at different times and in the various planes illustrated in Figure 4. 


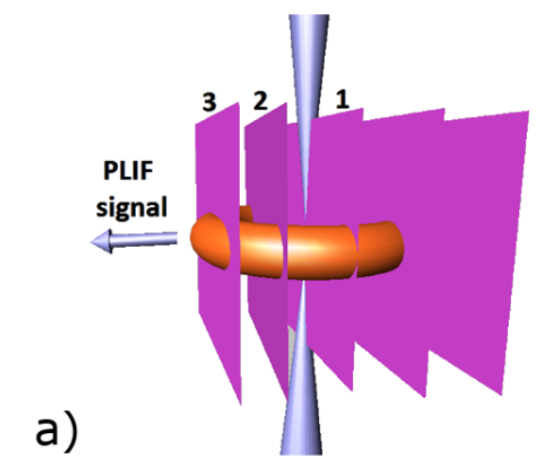

b)

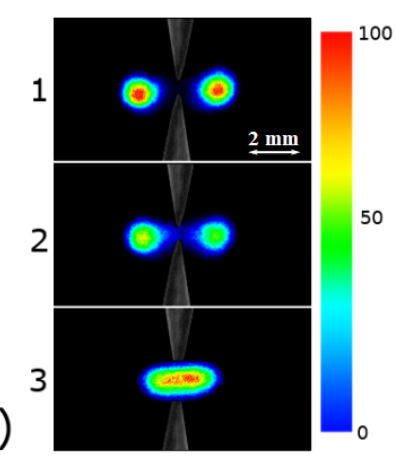

Figure 4. a) Schematic representation of the PLIF scans of the active medium in the discharge afterglow. b) PLIF signal obtained at $\mathrm{t}=100 \mu \mathrm{s}$ in the planes represented in Figure 4a. Plane 1 contains the axis of the electrodes. Planes 1, 2, 3 are each separated by $1 \mathrm{~mm}$. Interelectrode gap distance is $1 \mathrm{~mm}$.

The planes in Figure 4a represent successive positions of the laser sheet and the torus represents the active medium. Figure $4 \mathrm{~b}$ shows the corresponding PLIF images obtained in ambient humid air $(52 \%, 298 \mathrm{~K})$ for a spark initiated in a 2-mm gap by a single 10-ns pulse of $30-\mathrm{kV}$ amplitude. The PLIF images presented here were obtained $100 \mu \mathrm{s}$ after the discharge, with a camera gate of 100 ns. The PLIF images clearly confirm the toroidal shape of the active medium, with a characteristic diameter of $5 \mathrm{~mm}$.

The transformation of the spark channel into a torus is illustrated by the sequence of $\mathrm{OH}-$ PLIF images in Figure 5. In this case the laser sheet was in the plane containing the interelectrode axis (plane 1 in Figure 4a). The time stamp in each image corresponds to the time after discharge breakdown. The thin spark channel has an initial diameter of about $200 \mu \mathrm{m}$ and then transforms after about $50 \mu$ s into a torus of diameter $5 \mathrm{~mm}$. This happens due to the hot gas expansion in the radial direction and the influx of fresh gas from the electrode sides into the hot channel (see [Seydou2016, Castela2016]).

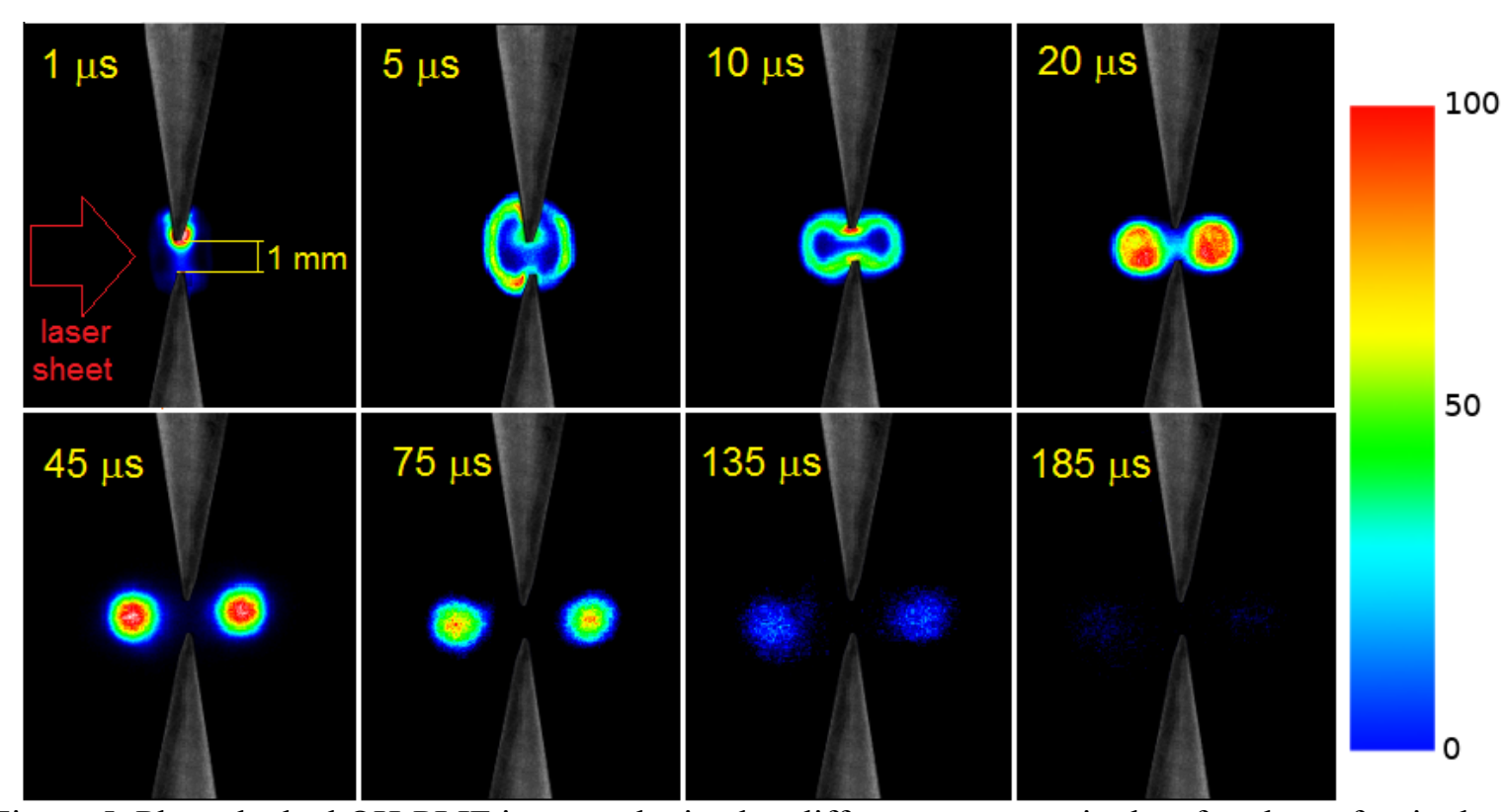

Figure 5. Phase-locked OH-PLIF images obtained at different moments in the afterglow of a single nanosecond spark initiated in ambient humid air. The discharge was initiated between pin electrodes by a 10-ns pulse of 30-kV amplitude. Interelectrode gap: $1 \mathrm{~mm}$. Energy deposited in the spark: $1.5 \mathrm{~mJ}$. Camera gate width: $100 \mathrm{~ns}$. 


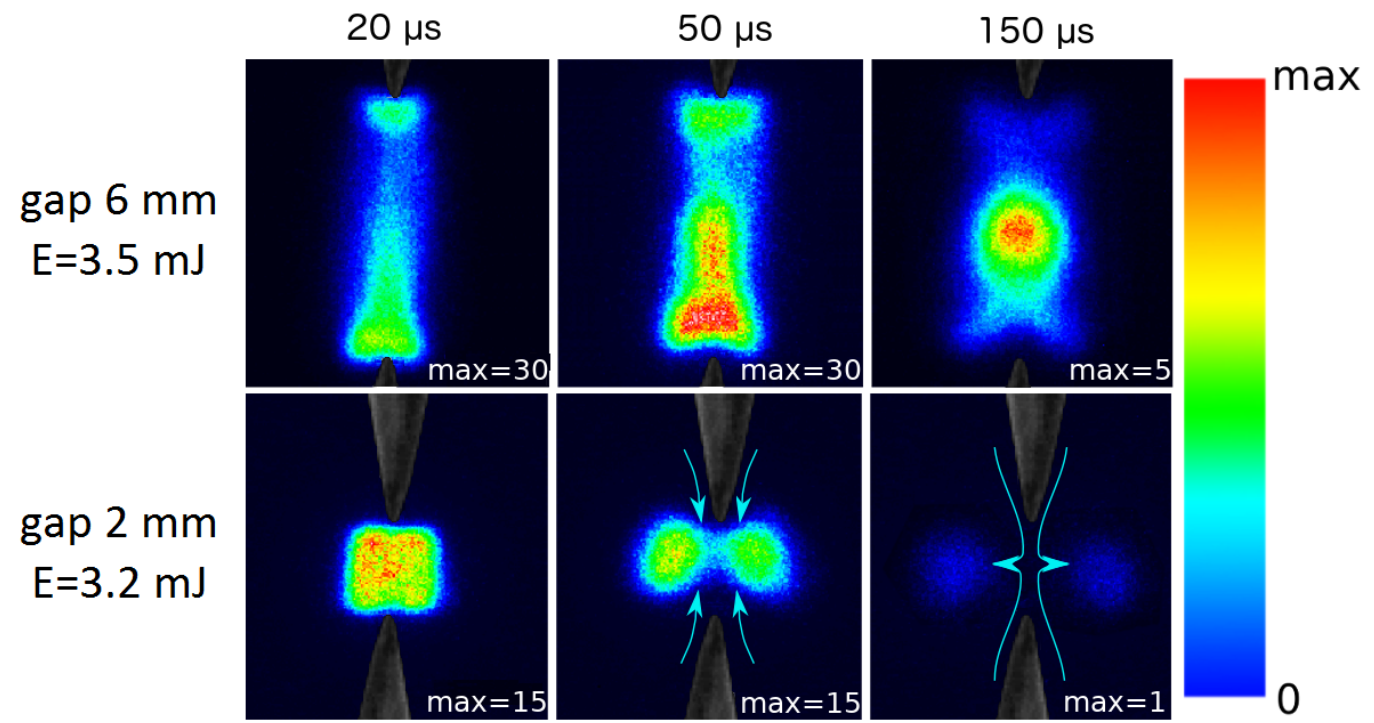

Figure 6. OH PLIF imaging at different instants after a single nanosecond spark. The discharge was initiated in ambient air by a pulse of 30-kV amplitude and 10-ns duration. The images were obtained for interelectrode gap distances of 2 and $6 \mathrm{~mm}$. The total deposited energy was approximately the same $(3.2$ vs $3.5 \mathrm{~mJ})$ in both cases. The camera gate was $100 \mathrm{~ns}$.

Figure 6 presents the comparison of OH PLIF images obtained for nanosecond sparks initiated in ambient air at two gap distances, $2 \mathrm{~mm}$ and $6 \mathrm{~mm}$. Both discharges were produced by a single voltage pulse of $10 \mathrm{~ns}$ and $30 \mathrm{kV}$. The total deposited energy was practically the same for these two cases (see Table 1), but the lineic energy was different $(1.6 \mathrm{~mJ} / \mathrm{mm}$ for the $2-\mathrm{mm}$ spark, and $0.6 \mathrm{~mJ} / \mathrm{mm}$ for the $6-\mathrm{mm}$ spark). Figure 6 shows that the recirculation only occurs in the case with the higher lineic energy.

To demonstrate that this result is not related just to the difference of interelectrode distances, we compare in Figure 7 two PLIF images obtained for two sparks with the same interelectrode distance but different deposited energies $(2.7$ and $4.8 \mathrm{~mJ})$. Both sparks were initiated in ambient air by a single $10-n s$ pulse of $30-\mathrm{kV}$ amplitude, with a 4-mm gap. The low-energy spark $(2.7 \mathrm{~mJ})$ was obtained with the $400-\Omega$ resistance in series with the electrodes (see Table 1). One can clearly see that the recirculation phenomenon only occurs for the high-energy spark $(4.8 \mathrm{~mJ})$.

a)

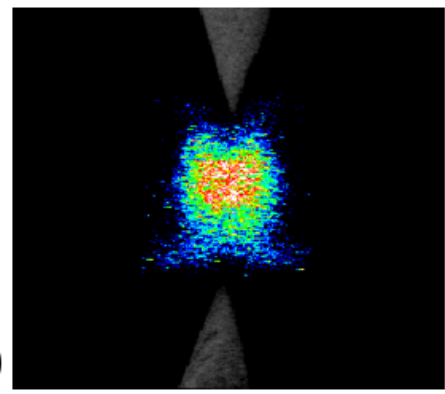

b)

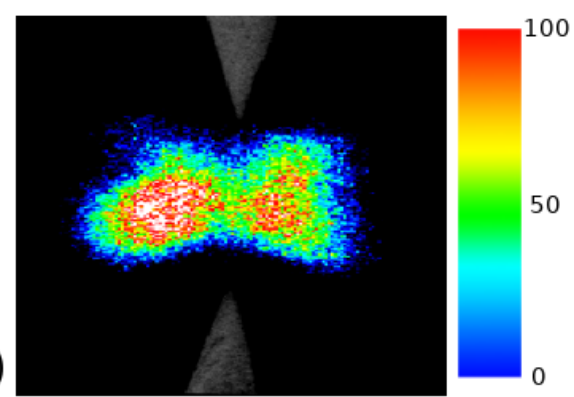

Figure 7. OH PLIF images obtained $100 \mu$ s after a single spark initiated by a nanosecond pulse in ambient air with a 4-mm interelectrode gap. Energy deposited in the plasma: a) $2.7 \mathrm{~mJ}$; b) $4.8 \mathrm{~mJ}$.

Another confirmation that the recirculation depends on the lineic energy is presented in Figure 8. These $\mathrm{OH}$ PLIF images were obtained $100 \mu \mathrm{s}$ after a single spark initiated in ambient air by a $10-\mathrm{ns}, 30-\mathrm{kV}$ pulse for various interelectrode distances but with the same lineic energy of about $1.5 \mathrm{~mJ} / \mathrm{mm}$ (see Table 1). The PLIF images presented in Figure 8 show that the recirculation occurs in all four considered cases (1,2,3, and 4-mm gap distances). 

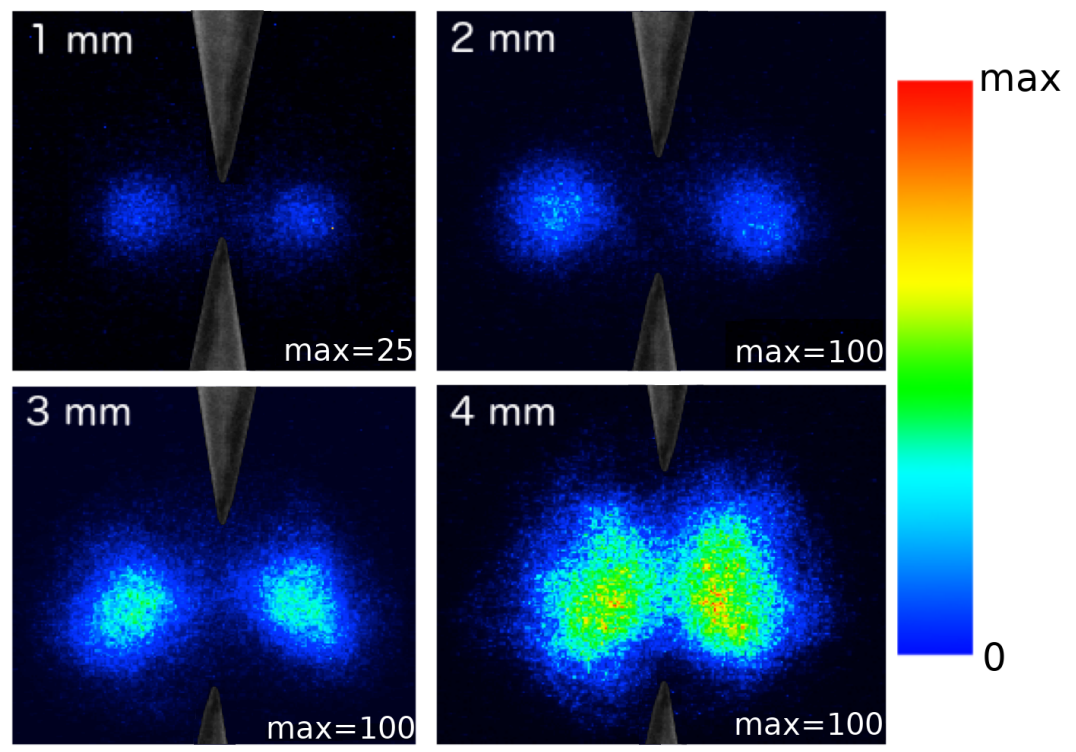

Figure 8. OH PLIF obtained in $100 \mu$ s after the discharge initiated at 1, 2, 3, and $4 \mathrm{~mm}$ gaps. All the sparks were initiated in ambient air by a single pulse of $U=30 \mathrm{kV}$ and $10 \mathrm{~ns}$ duration. The camera gate was $100 \mathrm{~ns}$. The lineic energy was approximately $1.5 \mathrm{~mJ}$ for all presented cases.

Furthermore, the dynamics of expansion of the active medium are practically the same for all considered interelectrode distances. Figure 9 presents the dependence of the characteristic dimension $d$ of the active medium versus time after breakdown for the cases presented in Figure 8. The characteristic dimension $d$ is defined in the figure. Quantitatively the border of the active medium was defined as the position where the LIF signal falls to $10 \%$ of its average value over the entire volume producing LIF. Although the choice of a $10 \%$ threshold may seem arbitrary, the gradients of LIF signal at the boundary of the active medium are so sharp that the actual value of this threshold has little impact on $d$.

One can see that from Figure 9 that the behavior of $d$ is very similar in all cases, except that the characteristic dimension at early times increases with the gap distance.

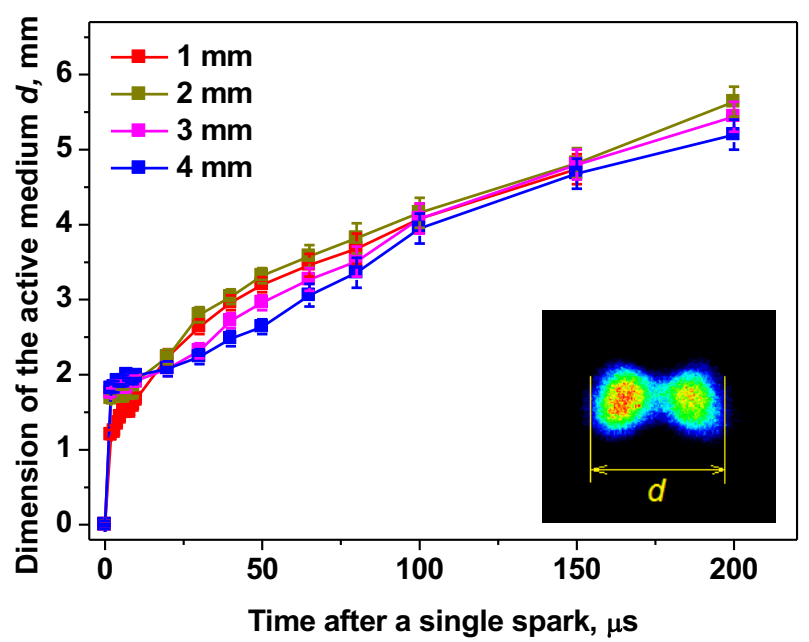

Figure 9. Evolution of the active medium dimension, $d$, after a single spark discharge initiated in ambient air by a pulse of 30-kV amplitude and 10-ns duration. Interelectrode gap distance: 1, 2, 3 and $4 \mathrm{~mm}$. The lineic energy was approximately $1.5 \mathrm{~mJ}$ for all presented cases. The data presented here were extracted from OH PLIF images. 
It should be noted that the recirculation phenomenon may be blocked if one of the electrodes is planar. The results of OH PLIF presented in Figure 10 were obtained for the discharge in ambient air initiated in pin-to-plane electrode geometry by a single $30-\mathrm{kV}$ pulse of 10-ns duration. In this case, fresh gas can enter near the tip of the pin electrode but is blocked on the side of the plane electrode. One can also see that the expansion of the active medium is stronger on the side of the pin electrode.
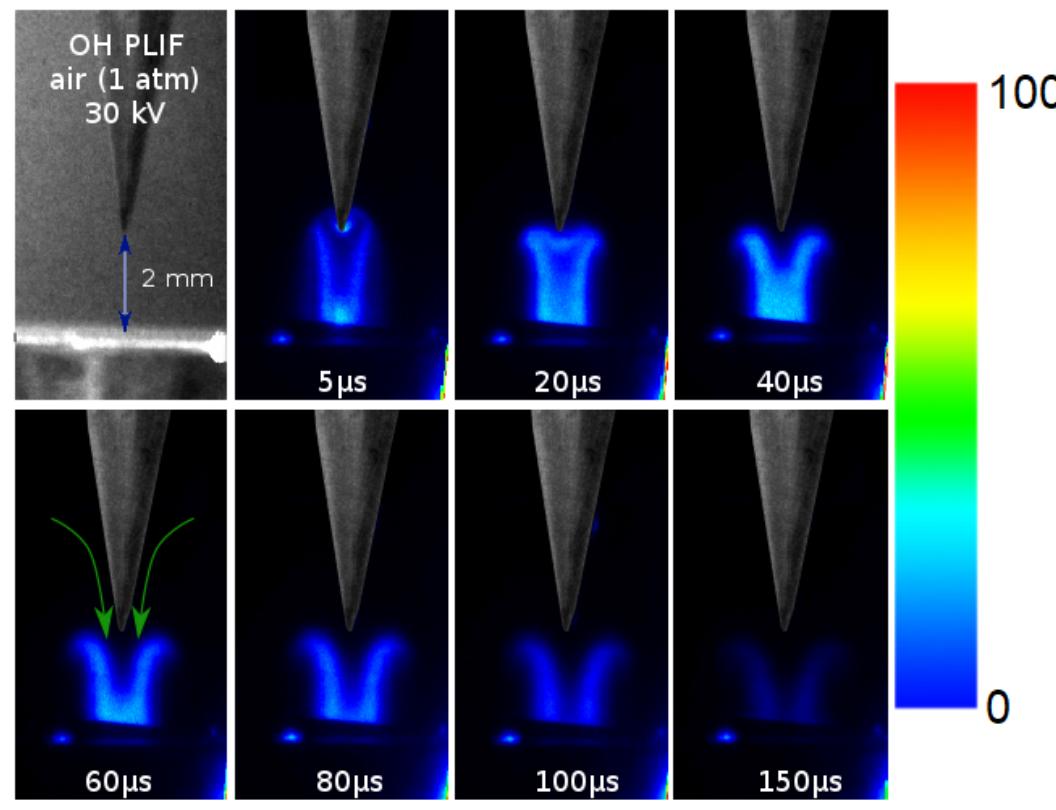

Figure 10. PLIF signal of $\mathrm{OH}$ for the discharge initiated in pin-to-plane geometry of the electrodes. The distance between the pin tip and the plane (on the bottom) is $2 \mathrm{~mm}$. The discharge was initiated in ambient air by a $10 \mathrm{~ns}$ pulse of $U=30 \mathrm{kV}$.

\section{Comparison of nanosecond and conventional automotive sparks}

We demonstrated in [Stepanyan2017] that nanosecond repetitive sparks generate excited gas volumes that are significantly larger than those produced by conventional automotive sparks [Maly1984, Maly1979, Lacour2016] for the same total deposited energy. In this section the reason for such a difference is investigated.

Figure 11 presents the results of OH PLIF for a conventional AUDI spark and for a single nanosecond spark initiated in gaps of 1 and $3 \mathrm{~mm}$ in ambient air. One can see that the recirculation of fresh gases occurs for the ns spark but not for the conventional spark.

Figure 12 shows the expansion curves corresponding to the PLIF images presented in Figure 11 for the 3-mm-gap. The maximal dimension $d$ of the active medium is significantly larger for the nanosecond spark $(6 \mathrm{~mm})$ than for the AUDI spark $(4 \mathrm{~mm})$, and this maximum dimension is achieved much earlier with the nanosecond spark.

We now compare the dynamics of energy deposition in both spark discharges. The energy deposited by the AUDI spark during the first $200 \mu \mathrm{s}$ is at least $10 \mathrm{~mJ}$ for a $3-\mathrm{mm}$ gap at normal conditions [Lacour2016] versus $4.1 \mathrm{~mJ}$ for the nanosecond spark. This period of $200 \mu \mathrm{s}$ corresponds to the stage of hydrodynamic expansion of the active medium for both discharges (see Figure 12). Therefore, it would be reasonable to expect the expansion to be more significant for the conventional AUDI spark as it releases more energy into the plasma. 


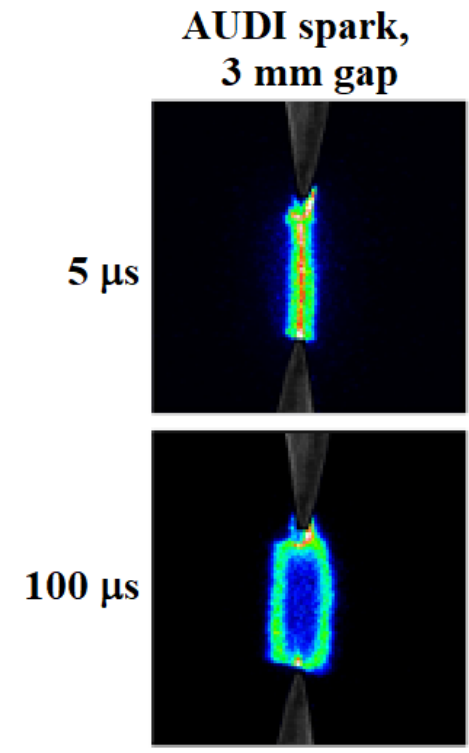

a)
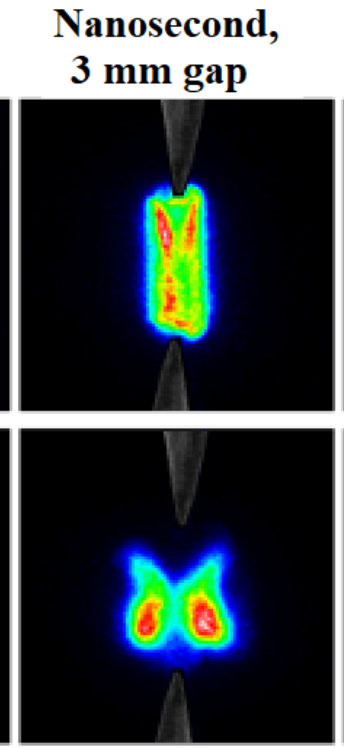

b)

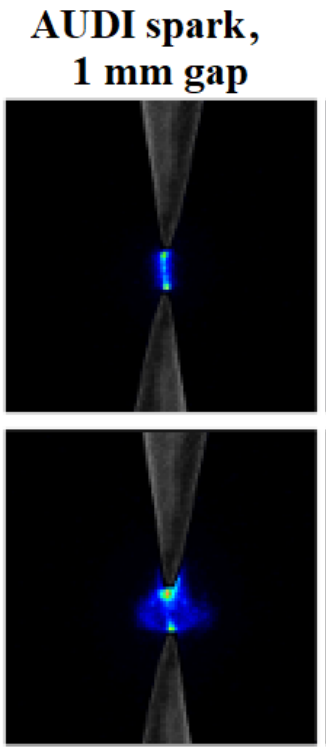

c)

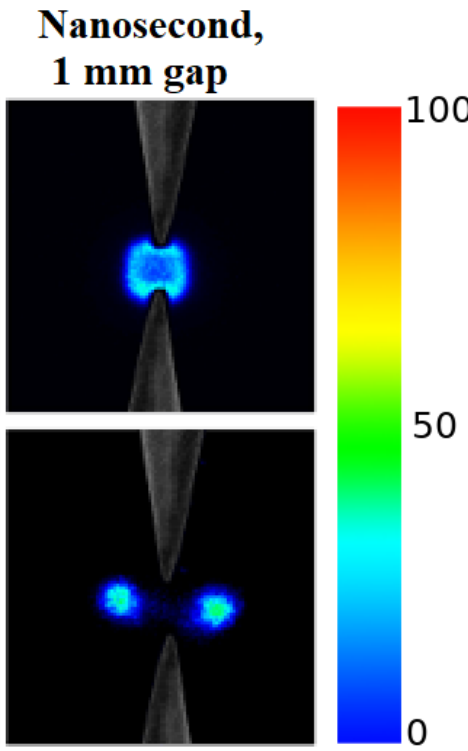

d)

Figure 11. OH PLIF images obtained for conventional automotive (AUDI) spark and single nanosecond spark obtained in ambient air at 5 and $100 \mu \mathrm{s}$ : a) AUDI spark, $3 \mathrm{~mm}$ gap; b) Nanosecond spark, $3 \mathrm{~mm}$ gap; c) AUDI spark, $1 \mathrm{~mm}$ gap; d) Nanosecond spark, $1 \mathrm{~mm}$ gap.

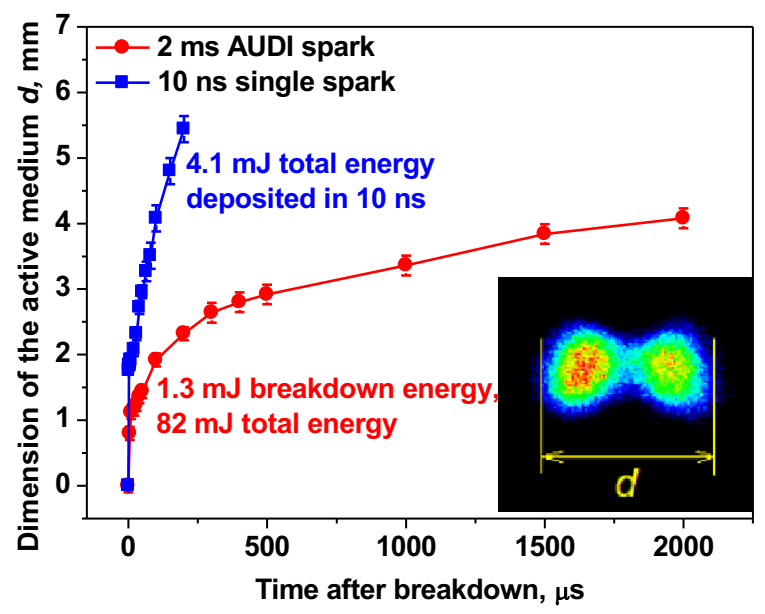

Figure 12. Evolution of the active medium dimension $d$ after breakdown. The discharge was initiated in ambient air for a 3-mm gap. The red circles correspond to the spark initiated by the AUDI spark system (2-ms duration) and the blue squares correspond to a spark initiated by a single pulse of 10-ns duration and 30-kV amplitude. Data extracted from OH PLIF images. For the nanosecond spark, the LIF and Schlieren signals become too weak to be detected after $250 \mu \mathrm{s}$.

However the experimental data demonstrate the opposite result. Meanwhile these results are in a perfect agreement with the data presented in [Maly1979]: the size of the volume excited by a conventional spark is entirely determined by the energy deposited during the breakdown stage of the spark and is not affected significantly by the subsequent glow stage.

According to [Lacour2016] the breakdown energy for a spark initiated by the AUDI spark in ambient air is $1.3 \mathrm{~mJ}$ for the $3-\mathrm{mm}$ interelectrode gap. This result is consistent with measurements presented by Maly [Maly1979]. For the nanosecond spark initiated at the same conditions, the breakdown energy is $4.1 \mathrm{~mJ}$, which is almost three times higher than for the AUDI spark. As one can see from Figure 12, the excited volume is larger in the case of the 
nanosecond spark owing to the higher breakdown energy, even though the total energy deposited by the AUDI spark is significantly higher than for the nanosecond spark: $82 \mathrm{~mJ}$ vs $4.1 \mathrm{~mJ}$.

These results demonstrate that, in order to increase the volume of active medium, it is necessary to introduce the energy in a short period, in accordance with the observation of Maly et al [Maly 1979]. In particular, a higher total energy input does not necessarily produce a larger volume of excited gas. It is better to introduce the energy over a period that is shorter than the typical hydrodynamic time. A confirmation of this statement can be found in the results presented in [Stepanyan2017], where we demonstrated that a burst of nanosecond sparks with the same total deposited energy as the AUDI spark produces a much larger excited gas volume than the AUDI spark.

\section{Hydrodynamic effects induced by a burst of nanosecond sparks}

An efficient way to enhance the active volume is to run repetitive nanosecond (submicrosecond) sparks with the highest available energy per pulse at frequencies on the order of tens of $\mathrm{kHz}$. In this case we observe a synergy between the hydrodynamic effects initiated by different sparks in the burst [Stepanyan2017], because the period between pulses is comparable with the typical expansion time of active medium.

We should mention that the Schlieren diagnostic has a significant advantage over PLIF when seeking to observe the hydrodynamic effects induced by a burst of nanosecond sparks. This is because the gas density perturbations (monitored by Schlieren) persist longer than the effective lifetime of $\mathrm{OH}^{*}$ (monitored by PLIF). Therefore, the analysis presented below is based on the Schlieren images.

In previous work [Stepanyan2017], we studied a burst of nanosecond sparks and showed that the dimension of the active medium after the burst grows linearly with the number of pulses, i.e. linearly with the amount of energy deposited in the plasma. However, the energy deposited in the plasma depends on many factors including the density and the nature of the gas; the shape, the amplitude, the duration and the frequency of the pulses; the interelectrode gap distance; and the electrical circuit of the high voltage pulse generator. Besides, some of the mentioned parameters can affect each another. For example, increasing the pulse frequency may change the gas composition and density before a new pulse arrives. Here we clarify the dependence of the active volume on the energy deposited in the plasma, on the interelectrode distance, and on the pulsing frequency. These are the parameters that can be most easily tuned for a given experimental setup, i.e. a combustor operating with a particular fuel and at a given pressure. As mentioned before, phenomena such as the formation of the ignition kernel, and the hydrodynamic effects induced by sparks tend to occur at the same time scale at pressures near or above $1 \mathrm{~atm}$.

Figure 13a presents Schlieren images obtained for a burst of 30 nanosecond sparks initiated in ambient air with an interelectrode distance of $1 \mathrm{~mm}$. The definition of the dimension of the active medium $d$ presented in Figure 13a is the same as for the LIF images. Schlieren images similar to the one presented in Figure 13a were used to extract the data presented further. Figure 13b presents the dependence of the characteristic dimension $d$ of the active medium versus the number of applied pulses for a burst of nanosecond sparks initiated in ambient air at $70 \mathrm{kHz}$ in a 1-mm gap. The energy deposited in each pulse in the burst is indicated for each experimental point. The characteristic dimension of the active medium $d$ is found to increase linearly with the number of sparks in the burst, i.e. with the energy deposited in the plasma. The red dashed line in the figure corresponds to the excited gas dimension for a conventional AUDI spark of $54 \mathrm{~mJ}$.

Figure 13c shows the dependence of $d v s$ the frequency of the applied pulses. In this case a burst of 30 sparks of $30-\mathrm{kV}$ amplitude (10 ns duration) was applied across a 3-mm gap in 
ambient air. The dimension of the active medium $d$ decreases with the frequency, from 15 to $10 \mathrm{~mm}$ in the $5-100 \mathrm{kHz}$ range. The data presented in Figure 13c, combined with the results of energy measurements [StepanyanAIAA2018], provide the average energy density inside the active medium for a burst of 30 sparks initiated in ambient air in a 3-mm gap. The active medium volume was determined by integrating the volume indicated by the Schlieren images assuming a symmetry of revolution. In the range $5-80 \mathrm{kHz}$, the average energy density inside the active medium is about $0.25 \mathrm{~mJ} / \mathrm{mm}^{3}$. At higher frequencies it increases up to $0.4 \mathrm{~mJ} / \mathrm{mm}^{3}$ at $100 \mathrm{kHz}$.

Finally, Figure 13d shows the dependence of $d v s$ the interelectrode gap distance for different frequencies of the burst of 30 sparks initiated in ambient air by 10 -ns pulses of 30 $\mathrm{kV}$. The largest active medium dimension $d$ is achieved for 3-5 mm interelectrode gaps in the considered range of frequencies.

a)

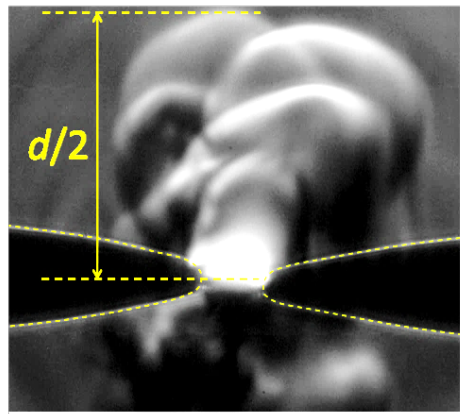

c)

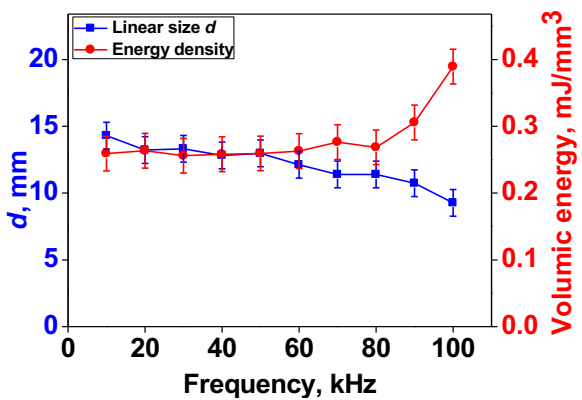

b)
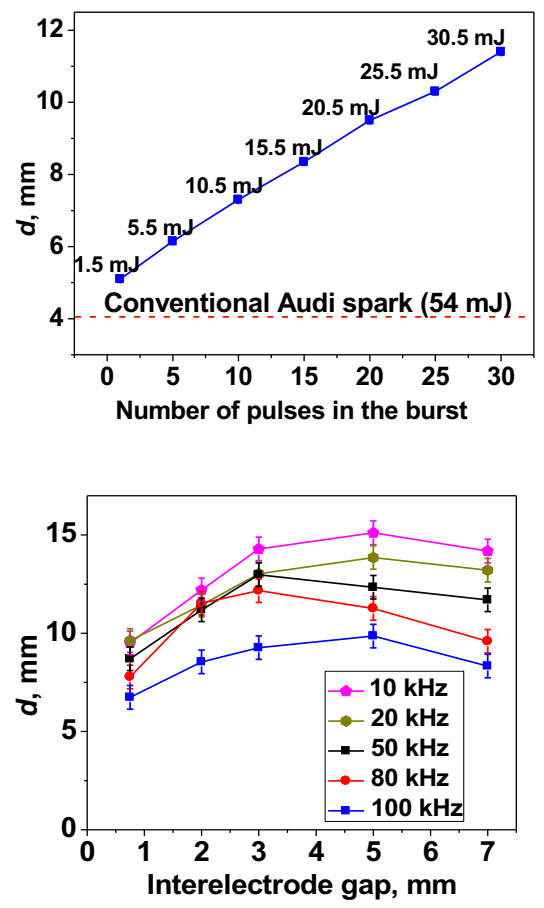

Figure 13. a) Characteristic Schlieren image of the active medium after 15 nanosecond sparks initiated in ambient air at $70 \mathrm{kHz}$. Interelectrode distance of $1 \mathrm{~mm}$; b) Dimension of the active medium $d v s$ number of pulses in a burst at $70 \mathrm{kHz}$ and 3-mm interelectrode gap; c) Dimension of the active medium $d v s$ burst frequency (blue squares) and corresponding energy density (red circles) for a 3$\mathrm{mm}$ interelectrode gap; d) Dimension of the active medium $d v s$ gap distance for different burst frequencies. All data correspond to discharges initiated in ambient air by $30-\mathrm{kV}$ pulses of $10-\mathrm{ns}$ duration.

\section{Conclusions}

In this article, the hydrodynamic effects induced by single or repetitive nanosecond sparks have been investigated in ambient air. The sparks studied here were initiated by pulses of 10ns duration, $30-\mathrm{kV}$ amplitude at frequencies up to $100 \mathrm{kHz}$.

The phenomenon of fresh gas recirculation after a single nanosecond spark has been studied with $\mathrm{OH}$ PLIF for various interelectrode gaps and energies deposited into the plasma. It was shown that this recirculation follows the expansion of the hot gas excited by the plasma. It was also confirmed that the recirculation phenomenon occurs only above a certain level of lineic energy deposited in the plasma, and that the expansion of the gas 
heated/excited by plasma increases with the lineic energy deposited in the nanosecond spark. Similar expansion dynamics were observed in various gaps $(1-4 \mathrm{~mm})$ with the same lineic energy deposited $(\sim 1.5 \mathrm{~mJ} / \mathrm{mm})$. We also showed that the gas recirculation does not occur when the geometry of the discharge electrodes blocks the entrance of the fresh gas into the interelectrode space, such as in the presence of a planar electrode.

The hydrodynamic effects induced by a single nanosecond spark and by a conventional automotive spark were compared. We showed that a nanosecond spark of $4.1 \mathrm{~mJ}$ excites a significantly larger volume than a conventional spark of duration $2 \mathrm{~ms}$ and energy $54 \mathrm{~mJ}$ (including $2 \mathrm{~mJ}$ in the breakdown stage). This difference is due to the fact that continuous energy addition maintains a hot central channel between the electrodes that prevents gas recirculation after the breakdown stage and therefore a larger expansion of the gas excited by the spark.

Repetitive nanosecond spark discharges provide a convenient way to introduce the energy in increments of a few $\mathrm{mJ}$ separated by periods of the order of tens of microseconds. In this case the recirculation phenomenon develops after each nanosecond spark and efficiently expands the size of active medium. The present research demonstrates that the characteristic dimension of the gas excited by a burst of 30 nanosecond arcs initiated in ambient air:

- grows linearly with each new spark, i.e. with the energy introduced in the gas

- varies from 10 to $15 \mathrm{~mm}$ for pulse frequencies in the range 5-100 kHz (3-mm interelectrode gap)

- varies from 7 to $15 \mathrm{~mm}$ for interelectrode gaps in the range $0.5-7 \mathrm{~mm}$ and frequencies from 10 to $100 \mathrm{kHz}$.

The parametric study shows that the typical volume of the gas excited by a few tens of nanosecond sparks is greater than $1 \mathrm{~cm}^{3}$. This excitation occurs during a few hundreds of microseconds, a period typically shorter than the ignition delay time.

The activation of significantly larger gas volumes using repetitive nanosecond sparks compared to conventional sparks is certainly a key for combustion improvement and particularly for the ignition and stabilization of lean flames.

\section{Acknowledgments}

This work was supported by the ANR FAMAC (Grant no. ANR-12-VPTT-0002) and PASTEC (Grant no. ANR-16-CE22-0005) projects. The authors thank Dr. Denis Packan (ONERA) for providing the Schlieren light source.

\section{References}

[Matveev2013] Matveev I. (2013) "Plasma Assisted Combustion, Gasification, and Pollution Control” Outskirts Press, Inc. Denver, Colorado, ISBN 978-1432786885

[Maly1984] Maly R. (1984) "Spark Ignition: Its Physics and Effect on the Internal Combustion Engine" Springer US, p. 91 (as a part of the book of Hilliard J., Springer G., (1984) "Fuel Economy in Road Vehicles Powered by Spark Ignition Engines" ISBN 978-14899-2279-3)

[Sher1992] Sher E., Ben-Ya'Ish J., Kravchik T. (1992) "On the birth of spark channels" Combustion and Flame 89 186-194

[Kossyi1992] Kossyi I., Kostinsky A., Matveyev A., Silakov V. (1992) "Kinetic scheme of the non-equilibrium discharge in nitrogen-oxygen mixtures" Plasma Sources Sci. Technol. 1(3) $207-220$ 
[Starikovskiy2013] Starikovskiy A., Alexandrov N. (2013) "Plasma-assisted ignition and combustion" Prog. Energy Combust. Sci. 39 61-110

[Castela2016] Castela M., Fiorina B., Coussement A., Gicquel O., Darabiha N., Laux C.O. (2016) "Modelling the impact of non-equilibrium discharges on reactive mixtures for simulations of plasma-assisted ignition in turbulent flows" Combustion and Flame 166 133147

[Bozhenkov2003] Bozhenkov S., Starikovskaia S., Starikovskii A. (2003) "Nanosecond gas discharge ignition of $\mathrm{H}_{2}-$ and $\mathrm{CH}_{4}-$ containing mixtures" Combustion and Flame 133(12) 133-146

[Wang2005] Wang F., Liu J.B., Sinibaldi J., Brophy C., Kuthi A., Jiang C., Ronney P., Gundersen M.A. (2005) "Transient plasma ignition of quiescent and flowing air/fuel mixtures" IEEE Trans. Plasma Science 33(2) 844-849

[Bellenoue2007] Bellenoue M., Labuda S., Ruttun B., Sotton J. (2007) "Spark plug and corona abilities to ignite stoichiometric and lean methane/air mixtures," Comb. Science Techn. 179(3) 477-496

[Singleton2011] Singleton D., Pendleton S., Gundersen M. (2011) "The role of nonthermal transient plasma for enhanced flame ignition in $\mathrm{C}_{2} \mathrm{H}_{4}$ /air" J. Phys. D: Appl. Phys. 44022001

[Adamovich2014] Adamovich I., Lempert W. (2014) "Challenges in Understanding and Development of Predictive Models of Plasma Assisted Combustion" Plasma Physics and Controlled Fusion 57(1) 014001

[Olsen1954] Olsen H., Edmonson R., Gayhart E. (1952) "Microchronometric Schlieren Study of Gaseous Expansion from an Electric Spark” Journal of Applied Physics 231157

[Kono1988] Kono M., Niu K., Tsukamoto K., Ujiie Y. (1988) "Mechanism of flame kernel formation produced by short duration sparks" Int. Symp. Combust. 221643

[Bane2014] Bane S., Ziegler J., Shepherd J. (2014) "Investigation of the Effect of Electrode Geometry on Spark Ignition" Combustion and Flame 162(2) 462-469

[Lo2014] Lo A., Cessou A., Boubert P., Vervisch P. (2014) "Space and time analysis of the nanosecond scale discharges in atmospheric pressure air: I. Gas temperature and vibrational distribution function of $\mathrm{N}_{2}$ and $\mathrm{O}_{2}$ " Phys. D: Appl. Phys. 47115201

[Seydou2016] Seydou A., Claverie A., Sotton J., Bellenoue M. (2016) "Experimental investigation of the effects of nanosecond repetitive pulsed (NRP) discharges on ignition of methane-air mixtures" 18th International Symposium on the Application of Laser and Imaging Techniques to Fluid Mechanics, Lisbon

[Lovascio2017] Lovascio S., Ombrello T., Hayashi J., Stepanyan S., A., Stancu G. D., Laux C.O. (2017) "Effects of pulsation frequency and energy deposition on ignition using 
nanosecond repetitively pulsed discharges" Proceedings of the Combustion Institute 36(3) 4079-4086

[Xu2016] Xu D., Lacoste D.A., Laux C.O. (2016) "Ignition of Quiescent Lean Propane-Air Mixtures at High Pressure by Nanosecond Repetitively Pulsed Discharges" Plasma Chem Plasma Process 36(1) 309-327

[Thiele2000] Thiele M., Selle S., Riedel U., Warnatz J., Maas U. (2000) "Numerical simulation of spark ignition including ionization" Proceedings of the Combustion Institute 28 1177-1185

[Ekici2007] Ekici O., Ezekoye O. A., Hall M. J., Matthews R. D. (2007) “Thermal and Flow Fields Modeling of Fast Spark Discharges in Air” J. Fluids Engineering 129 55-65.

[Castela2017] Castela M., Stepanyan S., Fiorina B., Coussement A., Gicquel O., Darabiha N., Laux C.O. (2017) "A 3-D DNS and experimental study of the effect of the recirculating flow pattern inside a reactive kernel produced by nanosecond plasma discharges in a methane-air mixture" Proceedings of the Combustion Institute 36(3) 4095-4103

[Stancu2008] Stancu G.D., Janda M., Kaddouri F., Pai D., Lacoste D. A., Rolon J. C., Laux C. O. (2008) "Investigation of the mechanism for rapid heating of nitrogen and air in gas discharges" $39^{\text {th }}$ Plasmadynamics and Laser Conference AIAA, Washington

[Rusterholtz2013] Rusterholtz D., Lacoste D., Stancu G. D., Pai D., Laux C.O. (2013) "Ultrafast heating and oxygen dissociation in atmospheric pressure air by nanosecond repetitively pulsed discharges" J. Phys.D: Appl. Phys 46464010

[Popov2001] Popov N. (2001) "Investigation of the mechanism for rapid heating of nitrogen and air in gas discharges" Plasma Physics Reports 27(10) 886-896

[Maly1979] Maly R., Vogel M. (1979) "Initiation and propagation of flame fronts in lean $\mathrm{CH}_{4}$-Air mixtures by the three modes of the ignition spark" Symposium (International) on Combustion 17(1) 821-831

[Do2010] Do H., Im S., Cappelli M., Mungal M.G. (2010) "Plasma assisted flame ignition of supersonic flows over a flat wall" Combustion and Flame 157 2298-2305

[Lefkowitz2018] Lefkowitz J., Ombrello T. (2018) "Reduction of flame development time in nanosecond pulsed high frequency discharge ignition of flowing mixtures" Combustion and Flame 193 471-480

[Stepanyan2017] Stepanyan S., Hayashi J., Salmon A., Stancu G. D., Laux C.O. (2017) "Large-volume excitation of air, argon, nitrogen and combustible mixtures by thermal jets produced by nanosecond spark discharges" Plasma Sources Sci. Technol. 26 04LT01

[Lacour2016] Lacour C., Lo A., Marrero J., Lefebvre F., Vervisch P., Cessou A., Lecordier B. "Characterisation of electric discharge in laminar flow with optical diagnostics" 18th International Symposium on the Application of Laser and Imaging Techniques to Fluid Mechanics, Lisbon, Portugal, 4-7 July 2016 
[Pai2008] D. Pai (2008) "Nanosecond Repetitively Pulsed Plasmas in Preheated Air at Atmospheric Pressure", Ph.D. thesis, Ecole Centrale Paris, France, can be downloaded at http://www.theses.fr

[Xu2013] Xu D. (2013) "Thermal and hydrodynamic effects of nanosecond discharges in air and application to plasma-assisted combustion" PhD thesis, Ecole Centrale Paris, France, can be downloaded at http://www.theses.fr

[Pai2010] Pai D., Lacoste D., Laux C.O. (2010) "Transitions between corona, glow, and spark regimes of nanosecond repetitively pulsed discharges in air at atmospheric pressure" Journal of Applied Physics 107093303

[StepanyanAIAA2018] Stepanyan S., Minesi N., Pannier E., Stancu G. D., Laux C. O. (2018) "Hydrodynamic effects induced by nanosecond repetitive pulsed discharges", AIAA paper 2018-0930, AIAA Aerospace Sciences Meeting, Kissimmee, FL, 8-12 Jan 2018 